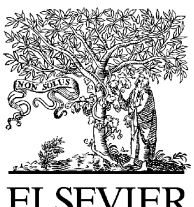

\title{
Under surface pressure sensing technique for the evaluation of contact stresses
}

\author{
R. Lupoi, F.H. Osman* \\ Department of Mechanical Engineering, University of Bath, Claverton Down, Bath BA2 7AY, UK
}

\section{Abstract}

In metal forming operations, the intensity and distribution of stresses at the tool/material interface have great influence on the pattern and mode of deformation. This is due to the effect of frictional forces, being resistive to material movements and hence affects material flow and the directionality of volume distribution of material inside die cavities. This paper explores the use of the pressure pin technique for the measurement of tool stresses at the interface between the tool and the deforming workpiece material. It also introduces a new experimental methodology for the measurement of stresses where the measuring device is concealed inside the tool and not subjected to the severe conditions at the interface surface. Such an arrangement prohibits the severe conditions at the interface from influencing or distorting the experimental readings and facilitates repeatability of results. Each tool containing measuring devices is split into two parts, one that includes the shape to be formed and house the pin heads (industrial die), while the second part includes the rest of the measuring column including the load cells. Using thermal insulation the second part can be protected from any thermal conditions at the interface when the tools are used in hot forming operations. Theoretical investigation of tool stresses is carried out using stress analysis. Experimental measurements of axial and radial stresses at different positions along the radius of an axi-symmetric billet are presented and compared with the theoretical results. Friction resistance and friction coefficient are also evaluated across billet surface and at different height reductions. Such an investigation gives better understanding of the interface conditions and parameters, which are among the essential input to the sophisticated modelling technology applied to metal forming processes.

(c) 2005 Published by Elsevier B.V.

Keywords: Pressure pin; Interface friction; Tool stresses; Contact stresses

\section{Introduction}

In metal forming operations, contact conditions, tool stresses and friction forces in particular, have great influence on the behaviour of material deformation and the mode by which material flow is directed. This applies to almost all contact forming processes, from simple geometry operations like compression, extrusion and rolling to complex forming patterns such as those produced by forging. Under industrial conditions, little is known about the local variation in contact properties and their effects on wear, surface cracks, tool deflection and tool damage. Surface stresses are also important in the tool design process with respect to tool definitions and features. Much has been achieved in the area of material-forming technology, concerning the

\footnotetext{
* Corresponding author.

E-mail address: f.osman@bath.ac.uk (F.H. Osman).
}

plasticity of metals, heat transfer, but to a limited extent when dealing with contact stresses frictional effects. When considering the nature of material deformation and the introduction of full numerical simulation to metal forming analysis, accurate description and characterisation of contact properties become necessary for obtaining a realistic solution.

Measurement of contact stresses using sensitive pins, usually incorporating strain gauge circuitry and mounted in the pressing tools, have been used to provide estimates of tool stresses at the interface in forming operations, including extrusion and rolling [1-3]. Piezo-electric and optical device sensing techniques have been used in the basic pin construction for signal detection [4-6]. Use of a three-pin system has been introduced, so as to evaluate frictional stresses and the coefficient of friction at the tool/material interface [7]. Those pins incorporated a zirconia rod in order to isolate the load cell from the severe thermal conditions at the interface in hot 
forming operations, where the workpiece temperature may reach $1000^{\circ} \mathrm{C}$.

Analytical and numerical methods have been used to predict tool and forming stresses during material processing. Also, an attempt was made to predict the effect of deformation on the microstructure of the final product $[8,9]$. In most of these methods a friction factor $(m)$ or a coefficient of friction $(\mu)$ is used, and assumed constant on all contact surfaces and throughout the process. However, in some applications an adaptive friction coefficient had been employed when simulating multi-stage forming processes [10]. The advances in such methods have greatly enhanced the scope of tool design and demonstrated that they are capable of predicting metal flow, cavity filling, stresses and strains. The accuracy of the results is directly influenced by the accuracy, or realism, of the process representation and its operational parameters.

This paper investigates the design of a new experimental set-up incorporating the pressure pin technique. The pin is concealed inside the die hence the drawbacks of exposure to the conditions at the interface are eliminated. Force components are measured at different angle to the surface of contact and compared with those obtained from the slab analysis technique. The proposed experimental set-up and the measuring pressure pins are adaptable to both cold and hot forming operations.

\section{Measurements at contact surface}

Metal forming is carried out by tools that are capable of withstanding high stresses, and when operating under hot conditions, the tools must also sustain their integrity and strength throughout the forming operation. In contrast, measuring devices operate under high level of sensitivity depending upon the tolerance and the response of electronic devices, gain and noise. In order to measure contact stresses along contact surfaces, sensors must be attached to the tool and have presence at such particular locations. Hence they would give true representation of the conditions at the tool/material interface. Some of the conditions at the interface are complex or severe for the use of sensitive devices, for example the application of lubricants and heat, the presence of scale, and material shearing actions are some of the elements that could seriously affect the measurements.

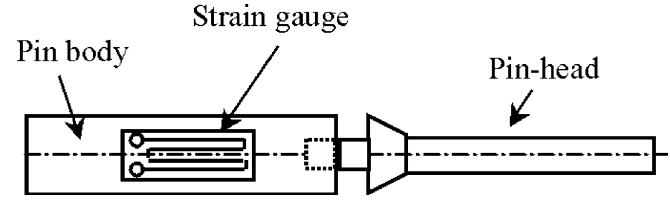

Fig. 1. Typical sensitive pin construction.

\subsection{Sensitive pins}

One of the methods that showed some resilience to surface conditions is the use of the pressure sensitive pins. They are made of similar material to that of the tool, therefore they would appear to be as an extension or part of the tool. Fig. 1 shows the shape and construction of a typical sensitive pressure pin. Because of its small size and its deformation characteristics, being similar to the tool, its presence is always assumed not to affect the measurements or the deformation process. The pin-head, transmits the load to the main body of the pin, which contains a load cell. Strain gauge bridge or small commercial load cells are commonly used with such pins.

\subsection{Sensitivity of measurement}

The pin must be free to move in order to accommodate any elastic strain in its axial direction. Therefore, there exist a gap between the pin-head and the hole in the body of the tool. In hot forming operation and in order to protect the measuring circuitry, the load cell may be insulated from the pin-head by introducing a layer of low thermal conductivity material, such as zirconia, between the two parts.

\subsection{Pin position and relative location}

The optimum position of the top surface of the pin-head is flush with the tool surface as shown in Fig. 2(a). The pin is usually adjusted to that position during the tool set-up, i.e. before the start of the forming operation. During the deformation process, there will be some relative displacement due to the elastic deformation of the pin and the tool. The pin may be pushed down into the tool by the deforming material as shown in Fig. 2(b) or penetrates through the material as shown in Fig. 2(c). This will, in someway, interfere with the defor-

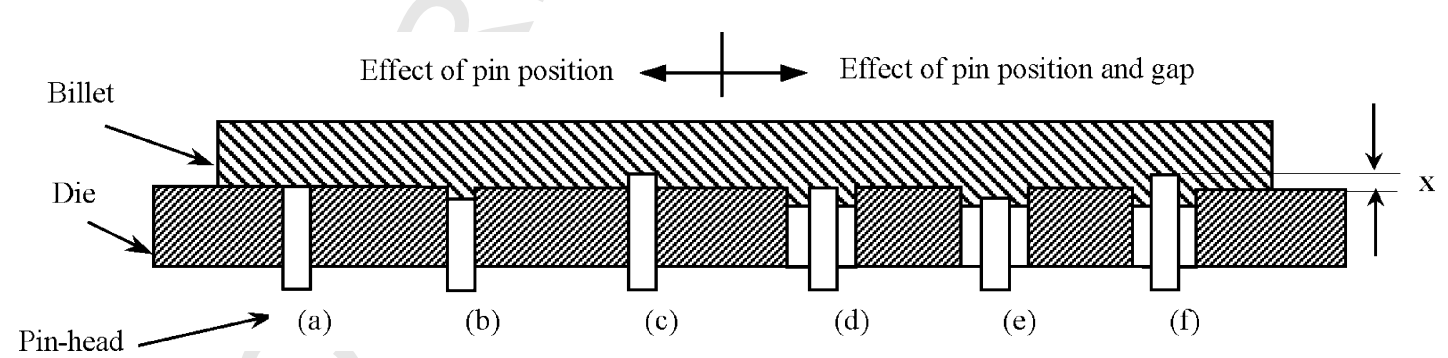

Fig. 2. Pin position and sliding gap relative to tool surface. 
mation mechanism at the contact surface and hence affect the measurements. Also, frictional resistance along the sides of the pin presents an unknown quantity as it depends on the narrowness of the gap around the pin-head and the presence of lubricant and scale in the vicinity of the pin-head. In addition, the deforming material might extrude through the gap between the pin-head and the die as shown in Fig. 2(d-f). This process is aided by the presence of the lubricant and the high local stress at the interface between the deforming material and the tool. Due to the loss of the pin flush position with the tool and the extrusion of the material around the pin-head, some accuracy would be lost as the pin-head would be severely constrained. Elementary tests were carried out to study the effect of the position of the pin-head relative to the interface on the stress measurements. Simple compression experiments were carried out on commercially pure soft aluminium $(99.5 \%$ ) billets of $25 \mathrm{~mm}$ diameter with the pin located in the die and coincides with the centre of the billet. The pin-head location relative to the tool surface, $x$, shown in Fig. 2 was varied from below to above the interface. A load of $450 \mathrm{kN}$ was applied to the billets to reduce the height from 30 to $8 \mathrm{~mm}$. Fig. 3 shows the pressure recorded by the pin relative to the average pressure acting on the whole surface of the billet at different pin heights. When the pin was positioned below the interface there has been signal delay until the material has extruded through the die hole and made contact with the pin-head. On the other hand, pins that positioned above the contact surface show an initial artificial jump in pressure, due to the initial impact with the deforming material. It is clear from the results shown in Fig. 3 that uncertainty, confidence level and accuracy of measurements depend on factors that relate to the positioning of the pin in the tool and the pin behaviour throughout the process of deformation. However, during the deformation process the position of the pin relative to the die surface will also vary according to the pressure acting on the pin-head. In other configurations, where the pin is positioned at an angle with the interface the pin section at the interface takes an elliptical shape and therefore, the pin column must contain an anti-rotation mechanism [7].

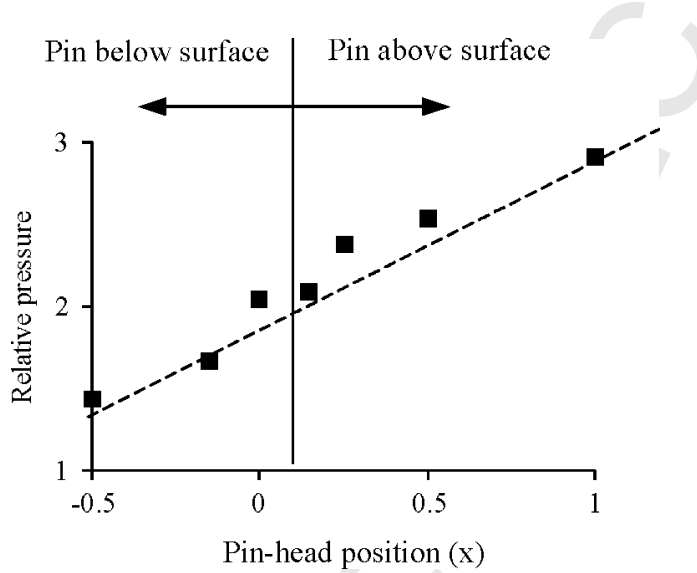

Fig. 3. Effect of pin-head location on measurements.

\section{A new strategy for tool design}

It is clear that the pin-head position relative to the surface of the tool varies during the deformation process and entrapment of the deforming material around the pin-head affects the accuracy of the measurements. An accurate measuring technique could be achieved if the pin-head is not positioned directly at the interface and not subjected to any relative displacement with respect to the tool. Such an arrangement would provide accurate permanent position for the pin and the pin could be considered as a truly integrated part of the tool material.

\subsection{Two-die system}

A tool integrated pin system is proposed with the added advantage that a two-die system is introduced; materialforming/industrial die and pin holders/measuring die. This will enable the use of vertical and oblique pins, with permanent contact with the tool. The material-forming die will include the geometry of the part to be formed and small holes for pin-head contact, these holes are to accommodate the locating of the pins but not used as guides, therefore they are of larger size than the $2 \mathrm{~mm}$ diameter pin-head. Fig. 4 shows the construction of the two-die system where the pin-head is embedded and concealed inside the forming tool. The pinhead top surface is located at a small distance, $1-2 \mathrm{~mm}$, from the interface, shown by the gap in Fig. 4 .

The pressure pin holding tool is of a simple geometry and includes through holes where pins are seated and locked in position when it is joined with the forming tool. Such an arrangement would make testing of industrial tools much easier, where no special tools need to be designed. The design of industrial tools involve CAD description of tools, adding stress testing locations at this stage will enable testing tool to be made concurrently and results validated at the same time with any process analysis. The design of the pin follows the typical sensitive pressure pin design. In these experiments, strain gauge pins are used and each pin consists of: pin-head, pin main body with an embedded strain gauge circuitry and a locking nut to ensure that the pin makes contact with the forming/industrial tool. The three parts are joined together through sliding sections to form a rigid measuring column, however, they can be separated easily. Changing a forming

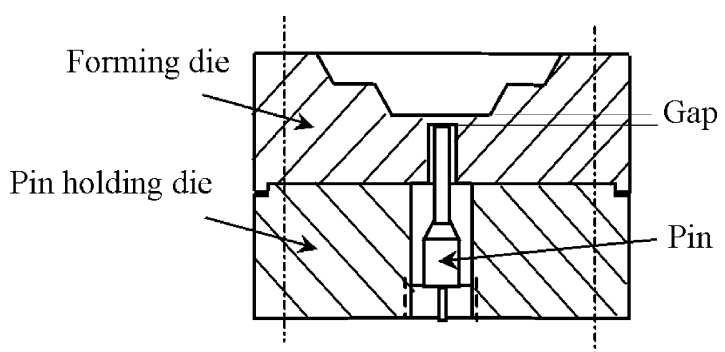

Fig. 4. Two-die system.
180 


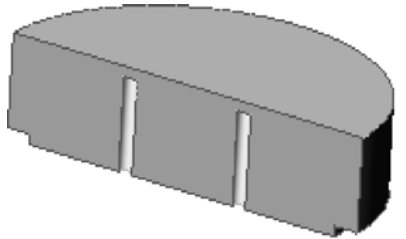

(a)

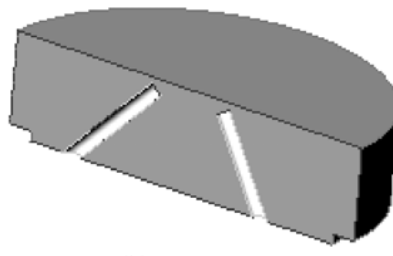

(b)

Fig. 5. Pin orientation in forming dies: (a) vertical pins and (b) inclined pins.

Pin $1 \Rightarrow F_{1}=A\left(P \cos \alpha_{1}+\tau \sin \alpha_{1}\right)$ $\operatorname{Pin} 2 \Rightarrow F_{2}=A\left(P \cos \alpha_{2}+\tau \sin \alpha_{2}\right)$

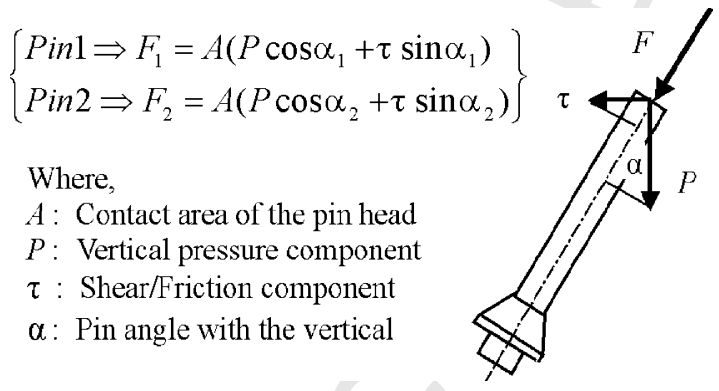

where $A$ is the contact area of the pin-head, $P$ the vertical pressure component, $\tau$ the shear/friction component and $\alpha$ the pin angle with the vertical.

\section{Slab analysis for compression}

Simple compression is utilised in this work in order to examine the suitability of the new die arrangement for interface pressure measurements. The sensitive pins are located $1 \mathrm{~mm}$ below the surface but they are calibrated to give the stress at the surface of contact. In the conventional compression test where a specimen is compressed axially between two parallel platens, friction occurring between the specimen and the tool causes bulging or barrelling at the outer surface of the workpiece during the deformation process. Analytical methods such as the classical slab method and the modified slab method give some insight into the distribution of surface stresses in the presence of friction. While the slab method neglects the influence of friction on the internal stress distribution the modified method postulates non-homogeneous deformation [11]. Results of both methods are significantly different for billets with height to diameter ratio greater than 0.5 .

\subsection{Stress system in simple compression}

Fig. 7 shows the system of stresses acting on a disc being compressed between two platens. Considering symmetry and equilibrium of forces in the radial and axial directions the equations for the pressure acting on the tool, $p$, and friction stress, $\tau$, become [12]:

$$
\begin{aligned}
& p=2 \tau_{k} \mathrm{e}^{(2 \mu / h)(R-r)} \quad\left(r_{\mathrm{s}} \leq r \leq R\right) \\
& p=\frac{2 \tau_{k}}{h}(R-r)+\frac{\tau_{k}}{\mu}[1+\ln 2 \mu] \quad\left(0 \leq r \leq r_{\mathrm{s}}\right)
\end{aligned}
$$

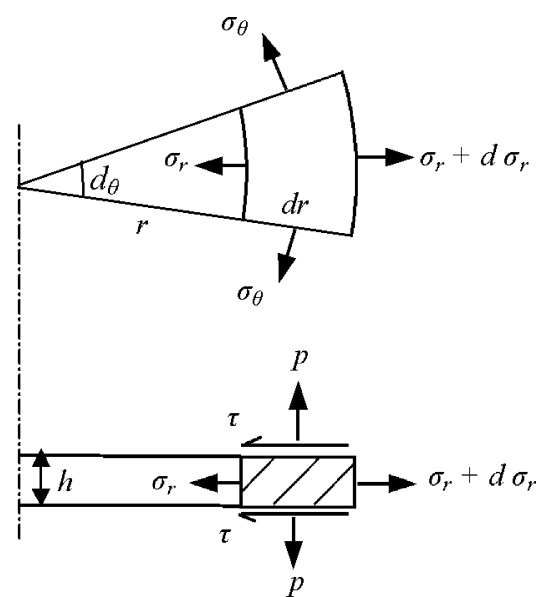

Fig. 7. Slab method stress system. 
and

$\tau=\mu p \quad\left(r_{\mathrm{s}} \leq r \leq R\right)$

$\tau=\tau_{k} \quad\left(0 \leq r \leq r_{\mathrm{s}}\right)$

where $R$ is the radius of the billet, $h$ the billet height, $\tau_{k}$ the material shear stress and $\mu$ the coefficient of friction at the tool material interface. The method assumes sticking and sliding zones, where $r_{\mathrm{s}}$ is the radius beyond which the material is allowed to slide in order to allow for radial expansion during the deformation process. Eqs. (1)-(4) represent the classical slab method of analysis, it assumes homogeneous deformation and a constant coefficient of friction. However, the implication of variable friction across the interface is relevant to this work.

\section{Experimental measurements of contact stress}

The new two-die arrangement design, where the pins are split between the industrial and measuring dies, were used to compress, under dry conditions, soft commercial $99.5 \%$ purity aluminium. Cylindrical specimens of $25 \mathrm{~mm}$ diameter and $30 \mathrm{~mm}$ height were used in all experiments. The material yield strength was found to be $100 \mathrm{MPa}$. The position of the pins is fixed inside the tool and therefore the stress distribution across the interface with the billet is obtained by using several billets. When inclined pins were used, flush to the contact surface [7], an anti-rotation mechanism was fitted to the pin column to prevent the pin elliptical cross-sectional area from being locked with the die surface. Such complication has been eliminated in the two-die arrangement where the pin-head is not subjected to any rotational force.

The calibration of pins was carried out by applying different loads to dies that were perfectly closed on a thin disk of aluminium. Also, a comparison has been performed between the load applied by the press and the total load obtained from the measured local distribution of normal tool stresses to ensure comparability, sensitivity and conformity of the pin calibration.

\subsection{Measurement of normal tool stresses}

Using the die with pins perpendicular to the contact surface, billets were compressed to a maximum of $400 \mathrm{kN}$. Eight billets were used for the measurement of normal local stress distribution across the deformed billets.

Fig. 8 shows the measurements of the normal pressure distribution at an early stage of the process $(20 \mathrm{~mm}$ billet height), a middle location $(15 \mathrm{~mm})$, and at the end of the process $(8 \mathrm{~mm})$. As the billet aspect ratio, height/diameter decreases, the stress in the centre of the billet increases and the difference between the stress at the centre of the billet and the stress at the end of the billet increases. The end stress value is very close to the material yield stress in all cases.

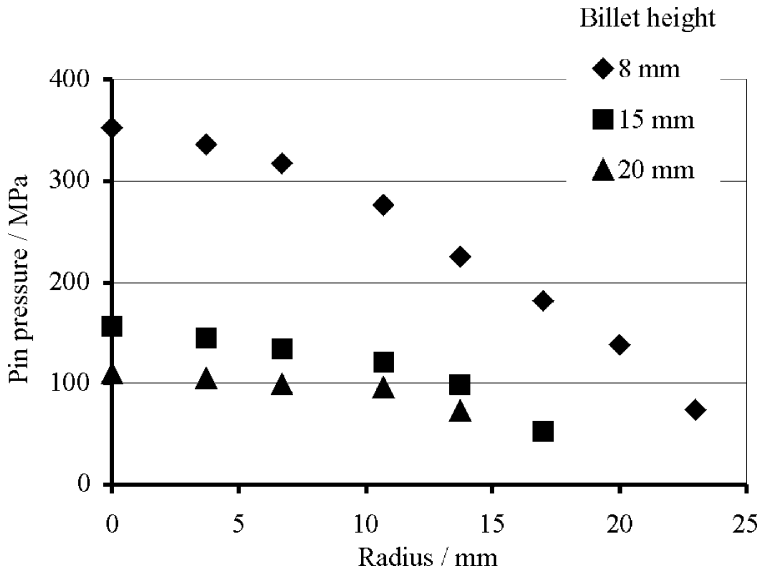

Fig. 8. Normal contact stress - vertical pins.

\subsection{Measurement of stress components at interface}

The stress at the interface may be composed of the vertical component that is normal to the interface, and the horizontal component acting along and parallel to the surface of contact, it may be expressed as 'friction stress'. Using the die with oblique pins, as shown in Fig. 5(b) the two components can be evaluated as function of the oblique angle, $\alpha$, as shown in Fig. 6. The two pins used in these experiments are those placed at $45^{\circ}$ and $30^{\circ}$ with the vertical axis. The measurements from both pins were obtained simultaneously during the deformation process for each billet. Similar to the experiments with the vertical pins a load of $400 \mathrm{kN}$ was applied to reduce billets to approximately $8 \mathrm{~mm}$ in height.

Figs. 9 and 10 show the distribution of the two components of stress at the interface, they are measured from $1 \mathrm{~mm}$ below the contact surface with oblique pins. The trend of the stress distribution is similar at both stages of the process. The directional inclination of the pin is significant when considering the material movement above the pin-head. The deformation force has a positive effect on the pin, i.e. pushing, if the pin column is opposing the forming force and a negative effect; i.e. dragging, if the material is moving away from the pin.

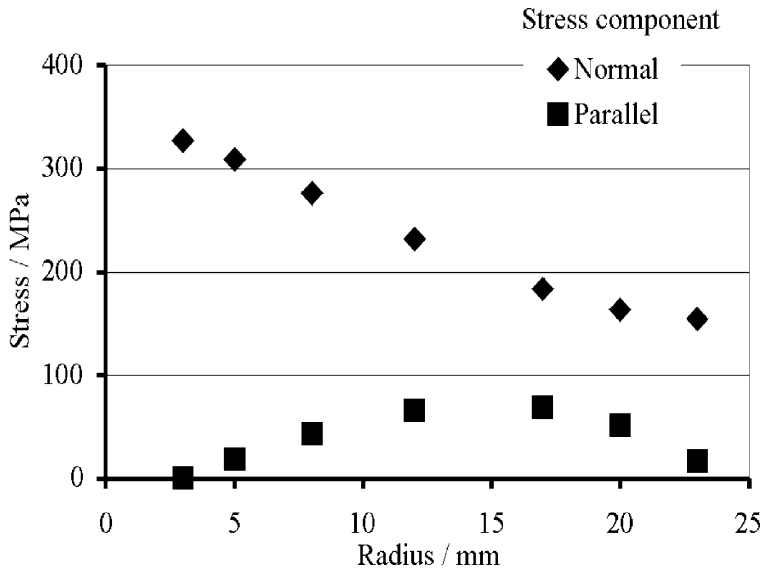

Fig. 9. Tool stresses at $8 \mathrm{~mm}$ billet height - oblique pins. 


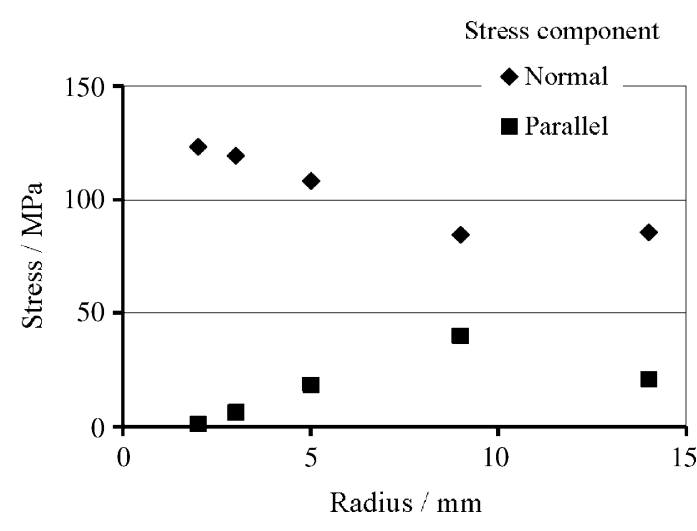

Fig. 10. Tool stresses at $20 \mathrm{~mm}$ billet height - oblique pins.

Hence, it is not practical to position the pin on the centre of the billet as half of its contact area will be pushed by the deforming material while the other half will be pulled. This is clearly evident from the results shown in Figs. 9 and 10 where there are no data points at dead centre. This of course does not apply to vertical pins where the force is always compressive.

\subsection{Evaluation of the forming coefficient of friction}

The normal and parallel measured components of stress given in Figs. 9 and 10 were used to evaluate the coefficient of friction at the interface for the two deformation stages. By definition the coefficient of friction, $\mu$, may be expressed by the following expression,

$\mu=\frac{\tau}{p}$

where $\tau$ is the friction stress and $p$ the normal stress at the interface. Fig. 11 shows the variation of the coefficient of friction across the surface of the billet at the early stage and final stage of the deformation. In both cases it steadily increases,

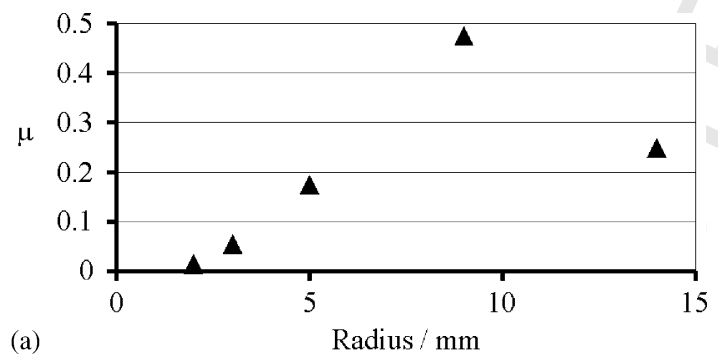

(a)

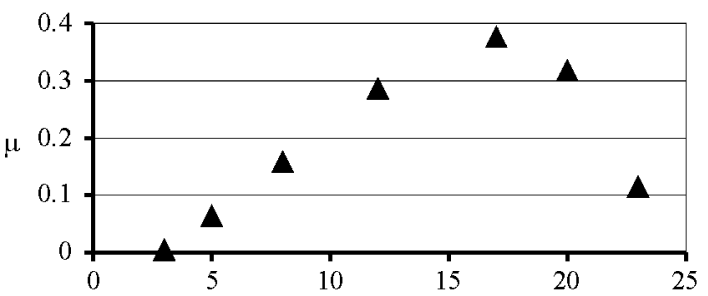

(b)

Radius / $\mathrm{mm}$

Fig. 11. Variation of coefficient of friction along the interface at (a) $20 \mathrm{~mm}$ and (b) $8 \mathrm{~mm}$ billet heights.

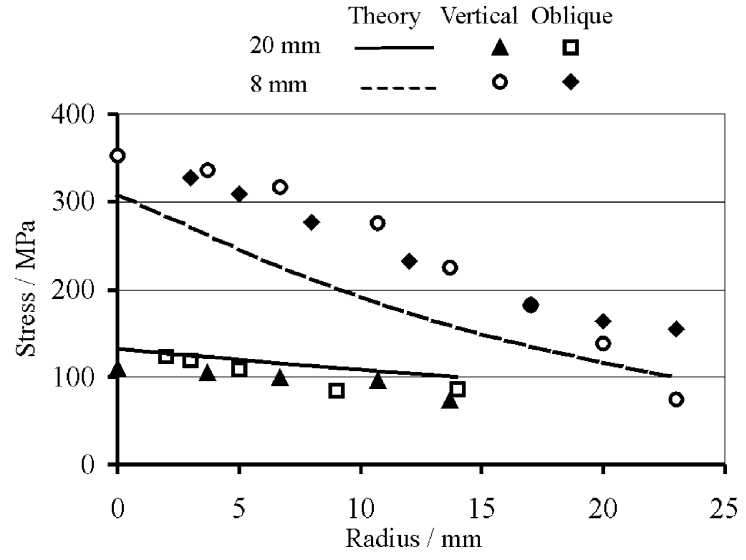

Fig. 12. Variation of coefficient of friction along the interface.

reaching a peak near $\mu=0.4$ then decreases towards the end of the billet.

354

\section{Integration of theory and experiment}

Knowledge of the state of stress and the shape of the friction function at the interface could be of significance in many material-forming processes. Also, it could be combined with analysis to give better prediction of metal flow and tool stresses. The slab analysis results are compared with those obtained from experiments and shown in Fig. 12. The results show a comparison between the distribution of the normal component of contact stress measured by the vertical pin, oblique pin and the slab analysis described in Section 5 with $\mu=0.2$. The results are shown for two stages of the compression process, 20 and $8 \mathrm{~mm}$ billet height respectively. The results are very comparable with respect to the trend where a maximum stress occurs at the centre of the billet, for the shorter billet. It is, however, important to incorporate friction as changing function in order to obtain realistic theoretical results.

\section{Conclusions}

The design of a metal forming process involves understanding of the mechanism of metal deformation and knowledge of the interaction between the workpiece material and the pressing tool. The sensitive pressure pin technique is well established as a method for direct measurement of high stresses, specially under sever frictional conditions.

A new die design methodology, incorporating embedded sensitive pressure pins were introduced. The method provides a set-up, where industrial dies could be used within laboratory conditions to evaluate contact stress at the interface between the tool and the deforming material. The tool design does not expose the pin to the severe conditions at the interface where it could suffer from significant inaccuracy. Vertical and oblique pins were tested in this work. The distribution of the 
normal and frictional components of stress across the contact surface in simple compression were obtained experimentally and hence used to evaluate coefficient of friction. The results showed that frictional characteristics vary along the interface and throughout the deformation.

The normal stress component was compared with the results obtained from the classical slab method of analysis, the comparison was favourable but it was noted that the slab method employs a constant friction factor and has many limitations. The method of using concealed sensitive pins reveals itself to be a direct measuring technique for the effective evaluation of contact stresses. It is therefore concluded that this technique is capable of providing experimental data for the simulation of complex metal forming operations.

\section{References}

[1] J. Frisch, An experimental study of metal extrusion at various strain rates, Trans. ASME 76 (1954) 599-606.

[2] C. Tuncer, T.A. Dean, Surface stress measurement techniques in metal forming, Int. J. Mach. Tools Manuf. 28 (4) (1988) 417428.
[3] J.G. Lenard, Measurement of friction in cold flat rolling, J. Mater. Shaping Technol. 9 (3) (1991) 171-180.

[4] I.M. Cole, D.H. Sansome, A review of the application of pin load-cell pressure measurement techniques to metal deformation processes, in: Proceedings of the Ninth MTDR Conference, 1968, pp. 271-286.

[5] P. Christensen, Computer aided design of forging dies using the upper bound technique, Ph.D. Thesis, Technical University of Denmark, Denmark, 1986.

[6] T. Yoneyama, Direct measurement of stress and heat between work and tool in metal forming, Ann. CIRP 39 (1990) 219-222.

[7] F.H. Osman, F. Potel, M.S. Loveday, Measurement of friction characteristics using three pin system, Key Eng. Mater. 177 (2000) 583-588.

[8] J.W. Brooks, T.A. Dean, Z.M. Hu, E. Way, Three dimensional finite element modelling of a titanium aluminide aerofoil forging, J. Mater. Process. Technol. 80 (1998) 149-155.

[9] R.H. Wagnor, J.L. Chenot, Fundamental of Metal Forming, Wiley, 1997.

[10] A. Behrens, H. Schaftstall, D. Landgrebe, Automated FEM simulation of complex multistage forging process by use of adaptive friction coefficient, in: Proceedings of the Fifth International Conference on Technology of Plasticity, Ohio, USA, 1996, pp. 393-396.

[11] O.M. Ettouney, K.A. Stelson, A modified slab method for axisymmetric upset forging, in: Proceedings of the 12th NAMRC, SME Conference, Iowa, 1984, pp. 133-140.

[12] A. Ghosh, A.K. Malik, Manufacturing Science, Ellis Horwood, 1986. 